\title{
mHealth impact on secondary stroke prevention: a scoping review of randomized controlled trials among stroke survivors between 2010-2020
}

\author{
Amelia K. Adcock ${ }^{1}$, Treah Haggerty ${ }^{2}$, Anna Crawford ${ }^{3}$, Cristal Espinosa ${ }^{4}$ \\ ${ }^{1}$ Cerebrovascular Division, West Virginia University, Morgantown, WV, USA; ${ }^{2}$ Obesity Medicine, West Virginia University, Morgantown, WV, \\ USA; ${ }^{3}$ Health Sciences Library, West Virginia University, Morgantown, WV, USA; ${ }^{4}$ Masters of Clinical and Translational Science Program, West \\ Virginia University, Morgantown, WV, USA \\ Contributions: (I) Conception and design: AK Adcock, T Haggerty, C Espinosa; (II) Administrative support: A Crawford; (III) Provision of study \\ materials or patients: A Crawford; (IV) Collection and assembly of data: All authors; (V) Data analysis and interpretation: AK Adcock, T Haggerty, C \\ Espinosa; (VI) Manuscript writing: All authors; (VII) Final approval of manuscript: All authors. \\ Correspondence to: Amelia K. Adcock, MD. 1 Medical Center Drive, Suite 9180, Morgantown, WV 26506, USA. Email: akadcock@hsc.wvu.edu.
}

\begin{abstract}
Background: A fundamental gap between clinical prevention and self-management awareness heightens the risk for stroke recurrence in approximately one-fourth of the highest risk stroke survivors annually. Secondary stroke prevention has the potential to be promoted by mobile health (mHealth) applications for effective real-world adoption of vascular risk factor mitigation. This scoping review aims to evaluate the impact of mHealth interventions and their effectiveness to reduce recurrent stroke rates among stroke survivors in randomized controlled trials (RCTs).

Methods: Scoping review in Ovid Medline, Cochrane Library, CINAHL, and Scopus for RCT literature employing mHealth among stroke populations published in English from 2010 to November 19, 2020. Small or pilot studies that included randomized design were included.

Results: A total of 352 abstracts met inclusion criteria; 31 full-text articles were assessed and 18 unique RCTs involving 1,453 patients ultimately fulfilled criteria. Twelve of 18 met the pre-defined primary outcome measure, including 2 studies evaluating feasibility. Eight of 18 only addressed recovery from index stroke deficits. Most outcomes focused on self-reported functional status, mood, quality of life or compliance with intervention; primary outcome was an objective metric in 4/18 (blood pressure readings, step number, obstructive sleep apnea support compliance). Intervention duration 2-12 months, with a median 9 weeks.

Conclusions: No high-quality evidence supporting mHealth applications to reduce recurrent stroke was found in this scoping review. Overall, most studies were relatively small, heterogenous, and employed subjective primary outcome measures. mHealth's potential as an effective tool for stroke stakeholders to reduce recurrent stroke rates has not been sufficiently demonstrated in this review. Future randomized studies are needed that explicitly evaluate stroke recurrence rate.
\end{abstract}

Keywords: mHealth; secondary stroke prevention; randomized clinical trial

Received: 27 June 2021; Accepted: 11 November 2021; Published: 20 April 2022.

doi: $10.21037 /$ mhealth-21-27

View this article at: https://dx.doi.org/10.21037/mhealth-21-27

\footnotetext{
$\wedge$ ORCID: 0000-0002-0723-3830.
} 


\section{Introduction}

At least one quarter of the approximately 800,000 strokes that occur annually in the United States are recurrent events, with the highest risk of recurrence or death from vascular events within the first 3 months after index stroke (1-3). Recurrent stroke is typically defined as a new focal neurological deficit otherwise meeting the standard definition of stroke that occurs at least $24 \mathrm{~h}$ following clinical stability of index stroke (4). Ninety percent of recurrent strokes are preventable through adequate control of vascular risk factors $(3,5)$, yet most studies continue to show ineffective management of stroke survivors' underlying hypertension, hyperlipidemia, diabetes, and physical activity (5). Thus, despite compelling evidence supporting secondary prevention, a fundamental gap prevails between real-world adoption of vascular risk factor mitigation strategies and those recommended by stroke prevention health guidelines (6).

Multiple barriers have been recognized to explain these grim statistics, including a lack of motivation and effective self-management models, sedentary behavior, untreated mood dysfunction, and inadequate stroke health education (7-13). As many as $40 \%$ of stroke patients in the United States were unable to verbalize at least one risk factor for recurrent stroke. This considerable lack of awareness hampers self-management efforts and reduces adherence to secondary prevention (14).

Although mobile health (mHealth) applications are related to a broad range of interventions and lack a universally accepted definition, the digital health division of the World Health Organization defines mHealth as the "medical and public health practice supported by mobile devices, such as mobile phones, patient monitoring devices, personal digital assistants, and other wireless devices" $(15,16)$. Mobile devices are facilitating the development of new telecommunication tools aimed at improving compliance in healthcare, and mHealth applications can be designed to provide real-time feedback to the user, allow individualized content and access to information about disease prevention, and facilitate social support (17). Therefore, mHealth may be an underutilized strategy in secondary stroke prevention.

Mobile applications can also improve compliance with treatment guidelines, provide disease risk/outcome information, and increase administrative efficiency (18). mHealth has also been reported to be advantageous in changing health behaviors, such as medication adherence, achieving exercise goals, and reducing anxiety to improve health outcomes in patients with chronic disease (19-21).

This study examines the available high-quality evidence as defined by randomized controlled trials (RCTs) of mHealth interventions and their ability to reduce recurrent stroke rates among stroke survivors. Maintaining healthy behaviors over the long term is one of the most challenging aspects to chronic disease, and mHealth technologies may represent a flexible and user-friendly solution to prevent stroke recurrence over the patient's lifetime. We present the following article in accordance with the PRISMA-ScR reporting checklist (available at https://mhealth.amegroups. com/article/view/10.21037/mhealth-21-27/rc).

\section{Methods}

We conducted a literature search for publications in Ovid Medline (2010-present), Cochrane Library (2010-present), CINAHL (2010-present), and Scopus (2010-present) for RCTs employing mHealth among secondary stroke populations. A search query was constructed in Medline around the concepts of mHealth (including mobile health, telehealth, telemedicine, and mobile and smartphone apps) and secondary stroke. We defined mHealth as the practice of medicine supported by portable diagnostic devices to provide services that facilitate health prevention and intervention via short-messaging-service (SMS), smartphone applications, handheld-imaging platforms, wearable devices, and miniaturized sensor-based technologies (22). Keywords and medical subject headings were both used in the query, and the search was then adapted for use in the other databases (see Appendix 1 for full Medline search strategy). All databases were searched from inception to November 19, 2020, with no limits applied to the search. We examined the reference lists of all included studies, and duplicates were removed.

The literature was screened for studies among adult patients with previous stroke that were published in the English language between 2010 and 2020. Studies prior to 2010 were excluded due to their lack of relevance in the technology-dependent field of mHealth. Studies were included if they reported the findings of RCTs employing mHealth strategies for secondary stroke prevention. Small or pilot studies were included provided they were of randomized design. Alternative (e.g., observational) study designs were excluded. Likewise, commentaries, letters to the editor, published protocol descriptions, 
nonhuman studies, and findings only published in abstract form, conference proceedings, or as a master's thesis or dissertation were also excluded. Interventions targeting healthcare professionals or that monitored patient data only were excluded.

Titles and abstracts were screened independently against the inclusion criteria by two authors (AA, TH). Each record was screened independently, with disagreements resolved through discussion and consensus. Full-text articles were screened by the authors (AA, TH) for final decisions regarding inclusion, with disagreement resolved by consulting a third author (CE). Information from each included article was organized using a structured form, including study design, pilot status, number of participants, study population, delivery agent, intervention, prespecified primary outcome, and results reported. The risk of bias was assessed by two authors independently (AA, TH). Publication bias of RCTs previously identified by Cochrane as bias risks are selection bias, performance bias, detection bias, attrition bias, reporting bias, among other less common causes (23).

\section{Results}

Our scoping literature review identified 352 abstracts, of which six were duplicates, two were written in languages other than English, and 43 were published prior to 2010. Two authors (TH, AA) reviewed the remaining 301 abstracts. The most common reason for exclusion was not an RCT (143 abstracts), followed by review article [69], RCT protocol only [34], studied intervention was not mHealth [18], or population studied was not stroke [6].

Thirty-one full-text articles were reviewed. Among those, 13 were excluded for the following reasons: duplication (2 abstracts), not an RCT [6], conference proceedings [1], and trial description only [4]. A total of 18 unique RCTs involving 1,453 patients fulfilled the criteria after full review (Figure 1).

The included studies were among diverse populations worldwide and included seven studies in Europe; three studies in the United States; two studies in each of China, Taiwan (China), and Africa (Ghana, Nigeria); and one study from each of Australia, Pakistan, and South Korea. Details of each study are included in Table 1. Eight studies enrolled participants with subacute stroke (as defined by $<6$ months of index stroke) (24-31), and 10 studied individuals with chronic stoke (21,32-40). Only two studies exclusively enrolled patients with ischemic stroke (IS)
$(25,29)$, with the remaining 16 studies including participants with a history of IS or hemorrhagic stroke. The length of the studies varied from two weeks to 12 months, with a median study duration of nine weeks. Interventions were diverse and included an mHealth interface of one-way communication tailored to the individual participants in four studies $(27,31,35,36)$, a robotic-assist device in one study (28), a preloaded app or virtual reality program for smartphones or tablets in four studies (25-27,30), an interactive intervention (as defined by within-participant group communication) in two studies $(21,38)$, a twoway audiovisual telehealth intervention in six studies $(24,26,30,32,33,40)$, and a combination of a preprogramed app and telehealth (two-way) interaction in 9 studies (24-26,28-30,32,33,40). Six studies incorporated some form of one-way interaction (e.g., SMS education or reminders) (31,34-38), and six incorporated some form of telemonitoring or objective biometrics $(21,24,29,31,33,39)$. Eight studies targeted stroke deficit recovery (cognitive, physical, behavioral medicine rehabilitation) (24-26,30,32,33,39,40), eight addressed vascular risk factors (exercise, blood pressure, obstructive sleep apnea, depression, health goals) (21,27,29,31,35-38), and seven rated overall functioning and other psychosocial scores as primary or secondary outcomes $(24,27,28,30,32,33,40)$. No RCT data with a primary or secondary outcome of reduced recurrent stroke rates was found.

The risk of bias in RCTs was rated as low by the by the Cochrane Collaboration tool; however, all of the reported studies were vulnerable to selection and performance bias inherent to any study involving mHealth. Many (8/18) $(21,25,26,30,31,33,36,40)$ of the reported studies were pilot or feasibility studies and thus were not powered for efficacy.

Three studies reported acceptable levels of feasibility $(25,31,36)$, and in 10 studies, the primary outcome was achieved $(21,24,25,29,30,32,33,35,36,39)$. The only study to employ a robotic-assist mHealth intervention failed to demonstrate improvement in the experimental group (28). However, the prespecified outcomes were quality of life (QOL)/self-rated depression scores, and both the control and experimental groups received the same amount of weekly human encouragement and engagement by study personnel. These interactions may have influenced participants' (regardless of robotic-assist device use) depression and QOL perceptions. Overall, the majority of studies achieved their primary outcome, and most demonstrated improvement in psychosocial domains (i.e., depression, QOL, and overall functional status). 


\section{Identification of studies via databases}

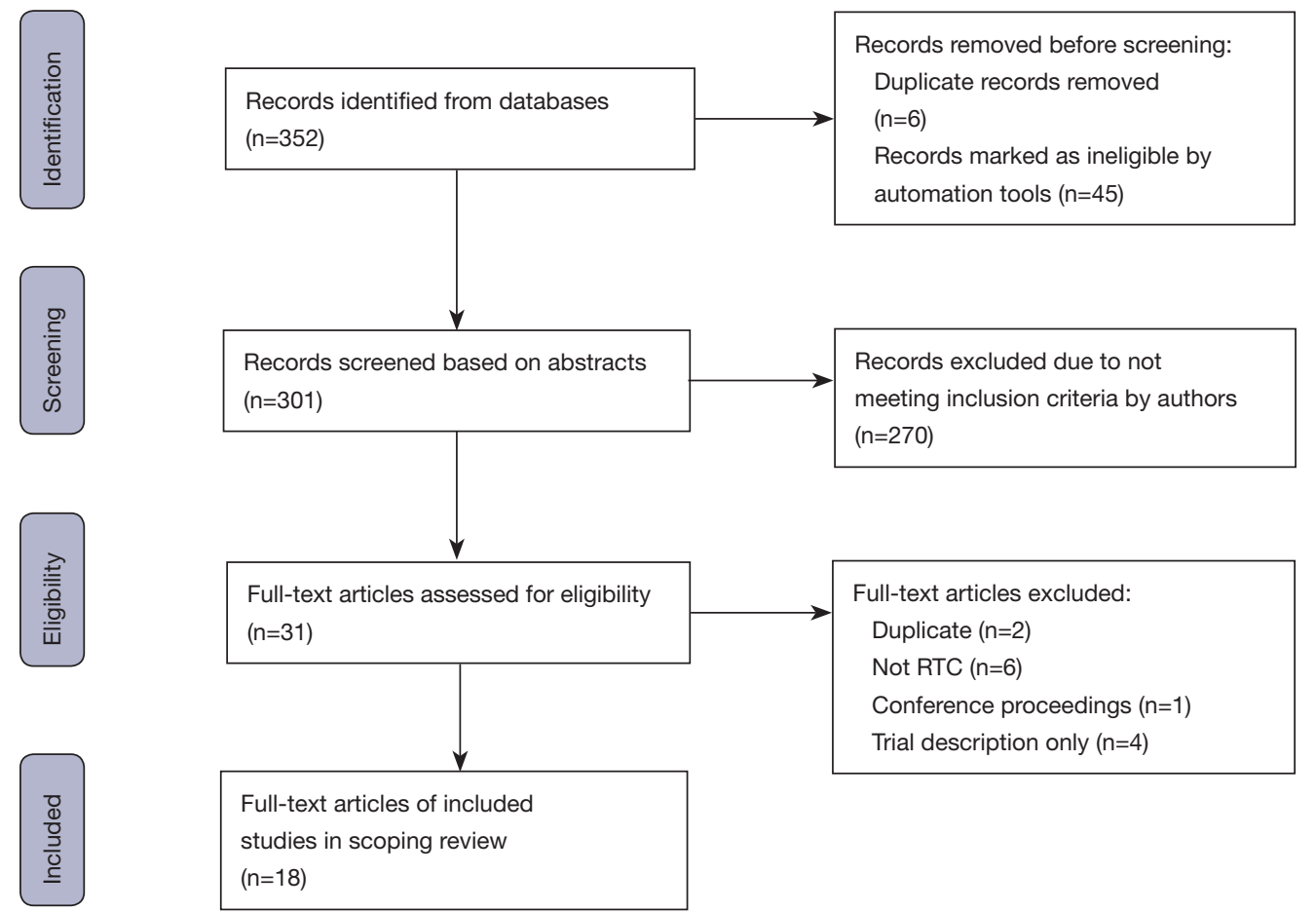

Figure 1 PRISMA 2020 flow diagram of search results.

\section{Discussion}

The burden of cerebrovascular disease is only expected to grow given the combination of our graying population and recent advances in acute stroke treatments leading to increased survival rates (41). Therefore, there is an urgent need to identify effective therapies to reduce recurrent stroke among those at high risk of additional cerebrovascular injury.

mHealth has been touted as a potential tool to reduce stroke recurrence by improving vascular risk factor profiles using widely available technology. However, it remains to be seen if this potential has been realized as demonstrated in robust clinical trials. The purpose of this review was to assess the application of mHealth interventions for secondary stroke prevention among published studies of randomized design.

Overall, this scoping review revealed very limited highquality evidence supporting mHealth applications to reduce the risk of recurrent stroke among stroke survivors. This finding echoes previous reviews published in $2016(42,43)$. Published evidence lags behind clinical implementation of an emerging tool such as mHealth, and rigorously designed large trials are likely ongoing (ClinicalTrials.gov Identifier: NCT04000971). However, it is still notable that no new studies were identified in the ensuing four years. Further, none of the included studies identified reduction in recurrent stroke rate as their primary outcome measure. Although improving vascular risk factors (e.g., decreasing blood pressure, addressing depression, treating sleep apnea, or increasing physical activity) can all be inferred as targeting recurrent stroke risk, most RCTs reported in this review identified explicit recovery from the index cerebrovascular event as the primary focus of their mHealth intervention. Among the studies focused on improvement in motor, speech, or cognitive deficits following stroke, most reported improvements in psychosocial outcomes in the experimental groups. Depression is an established independent risk factor for vascular events and death (44-46); therefore, strategies aimed at its mitigation are likely to contribute to an overall reduction in recurrent stroke $(47,48)$.

There was diversity in the types of interventions used for secondary stroke prevention utilizing mHealth. Many 


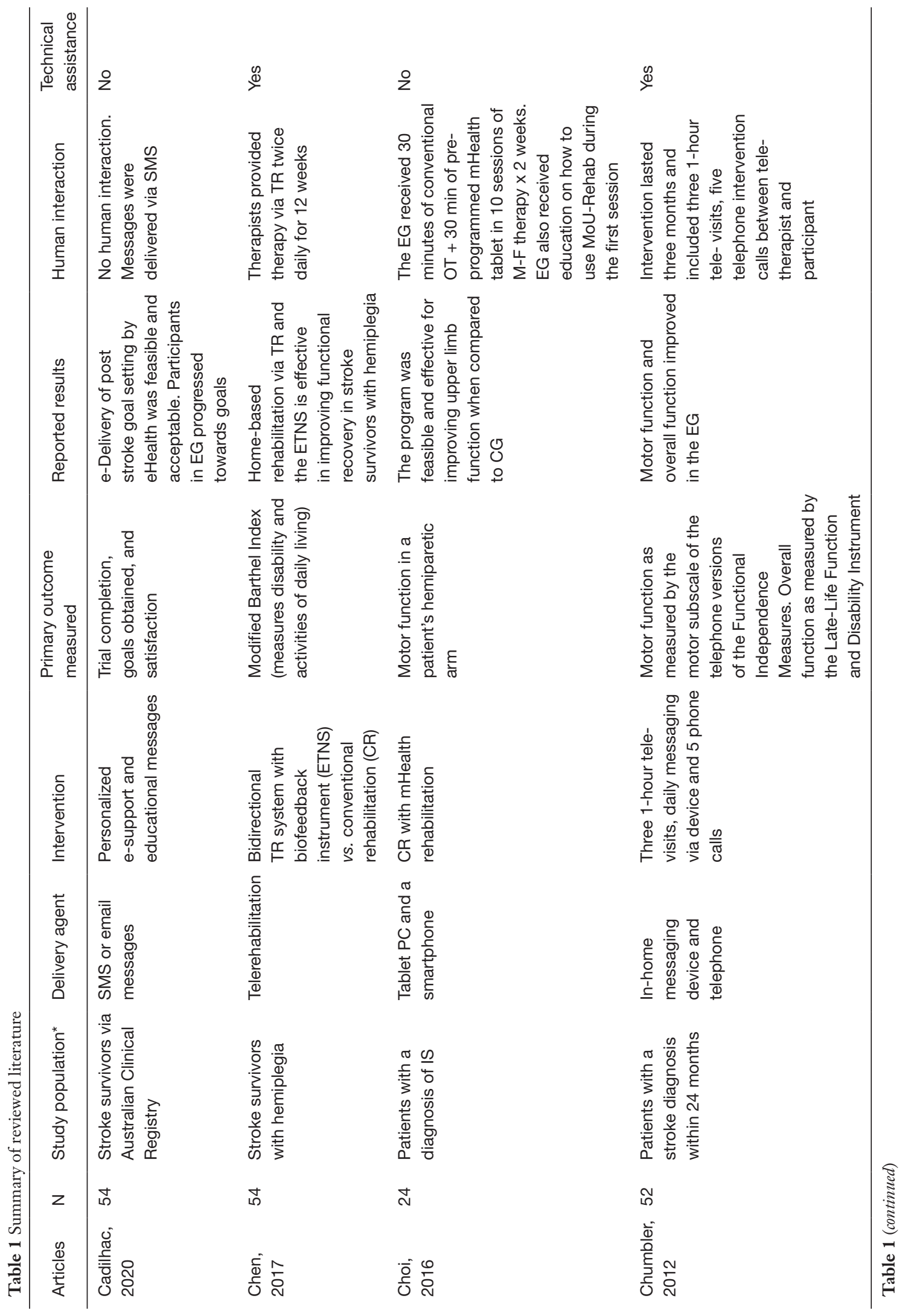




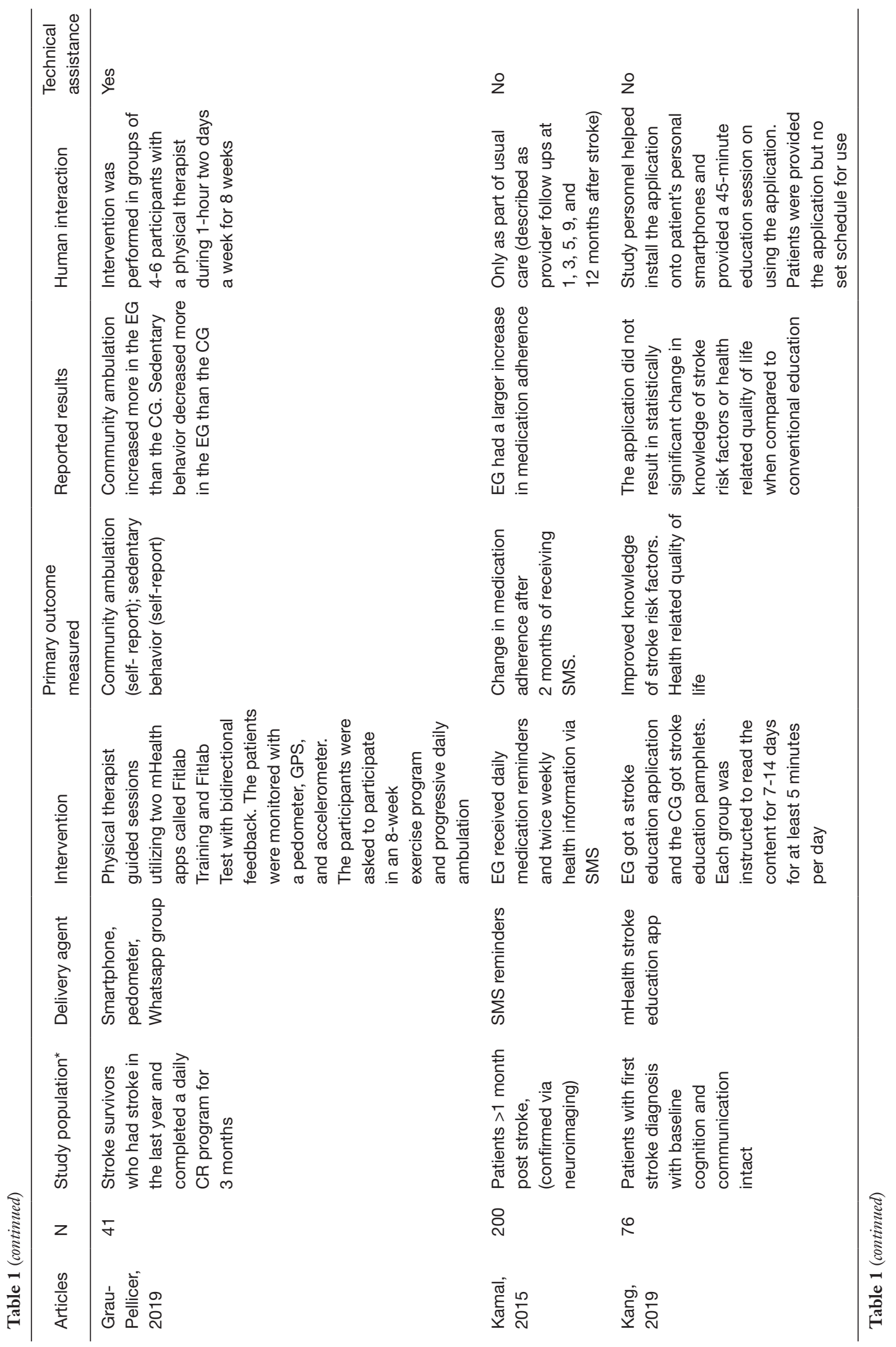




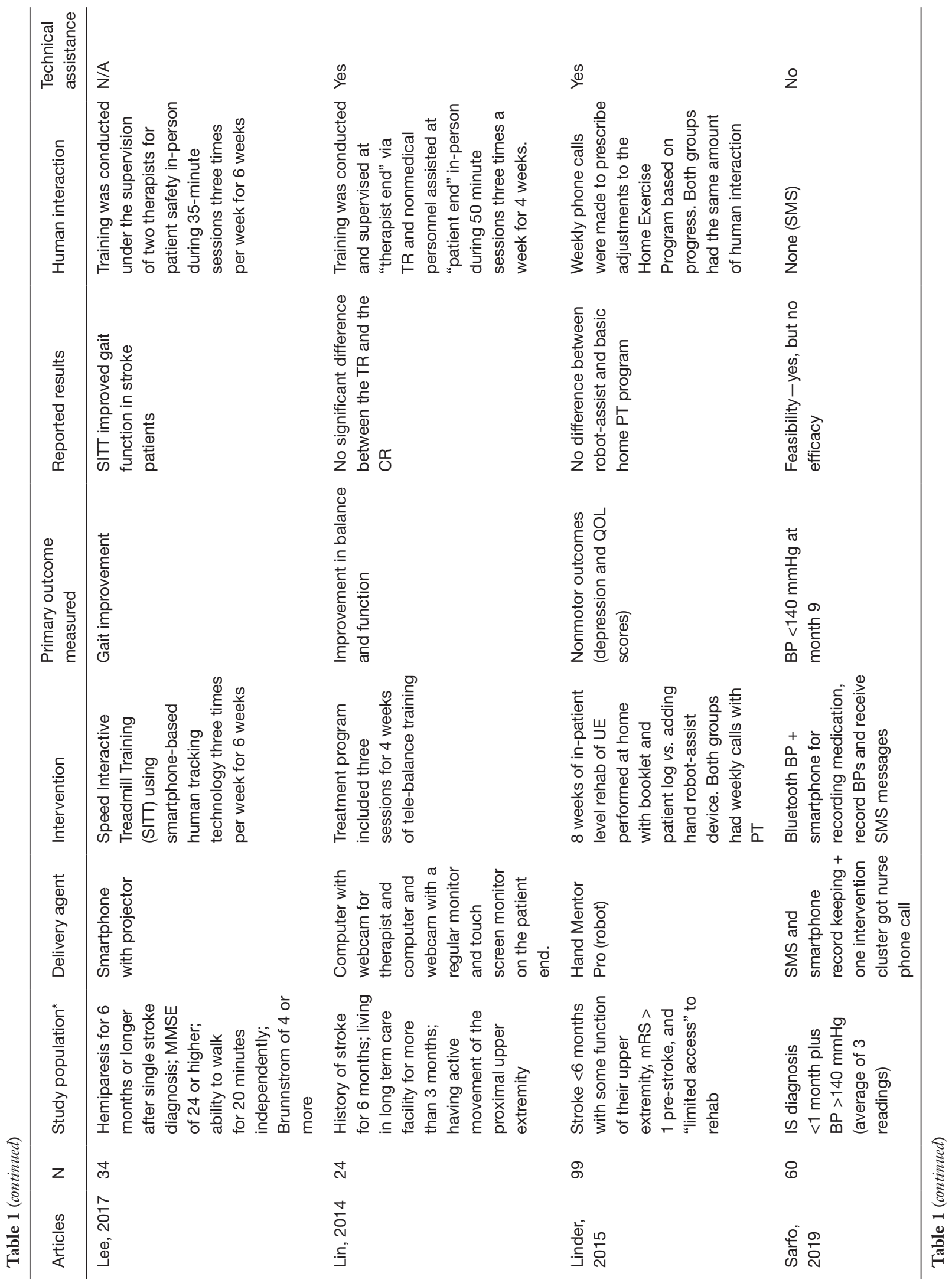




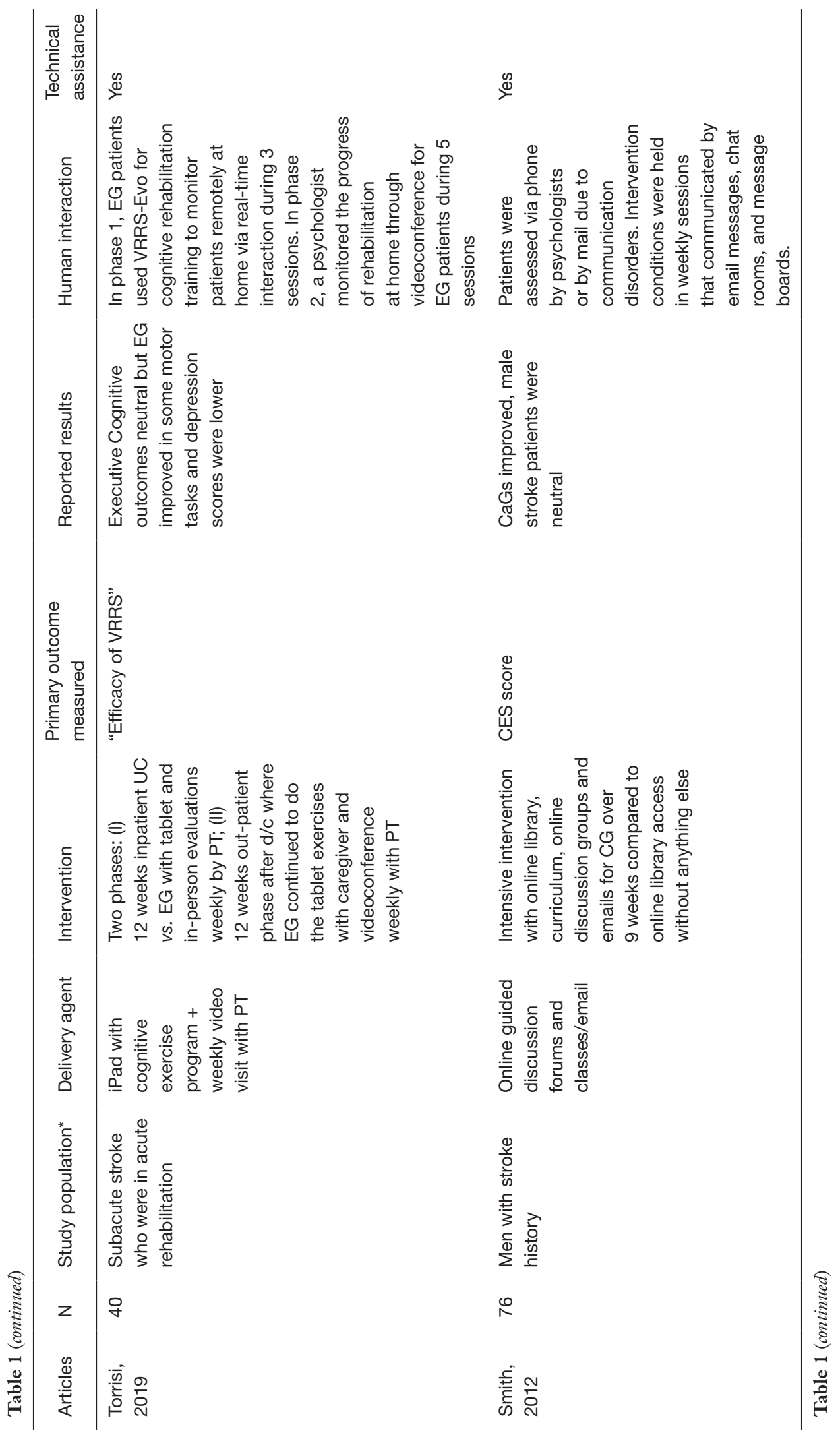




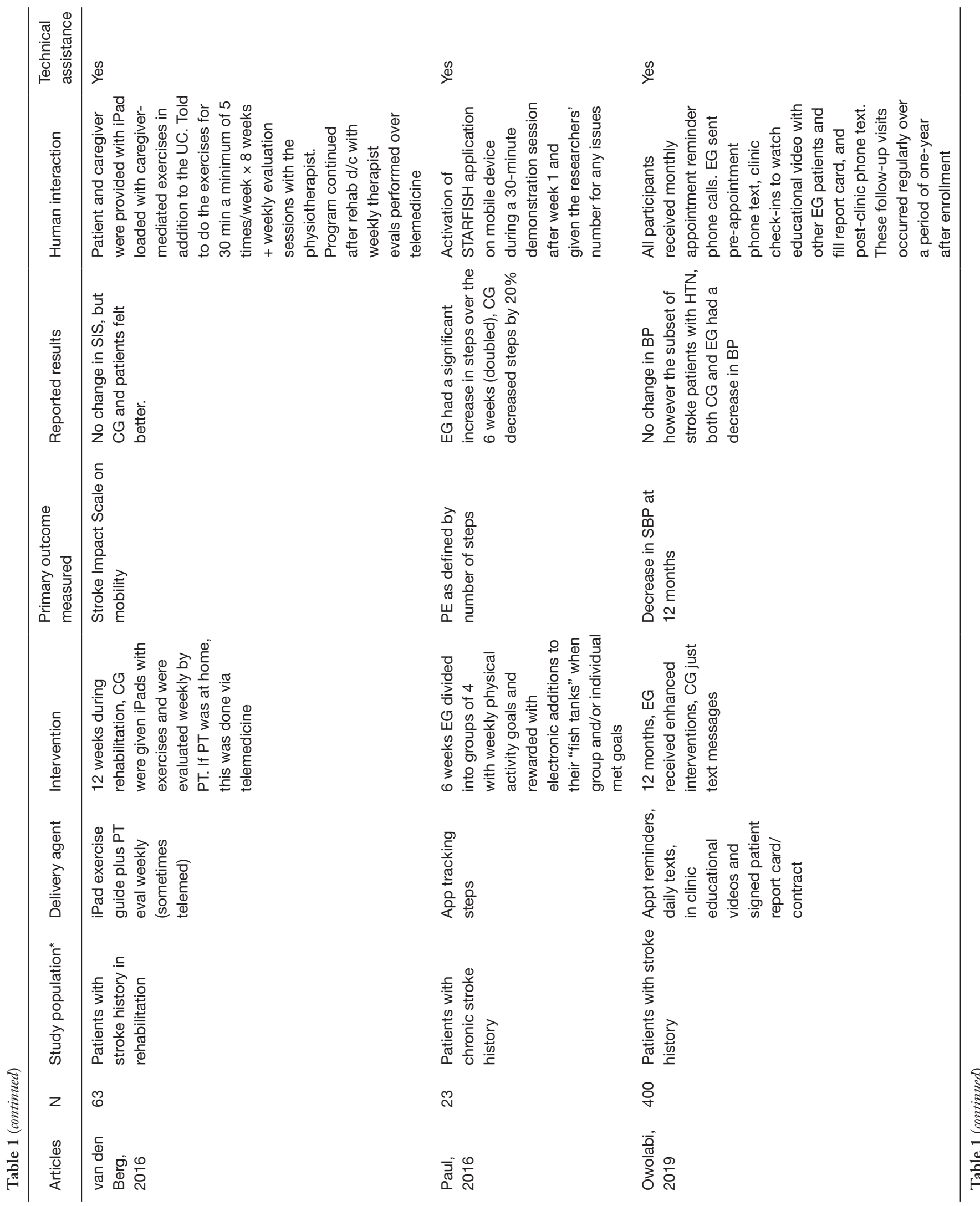




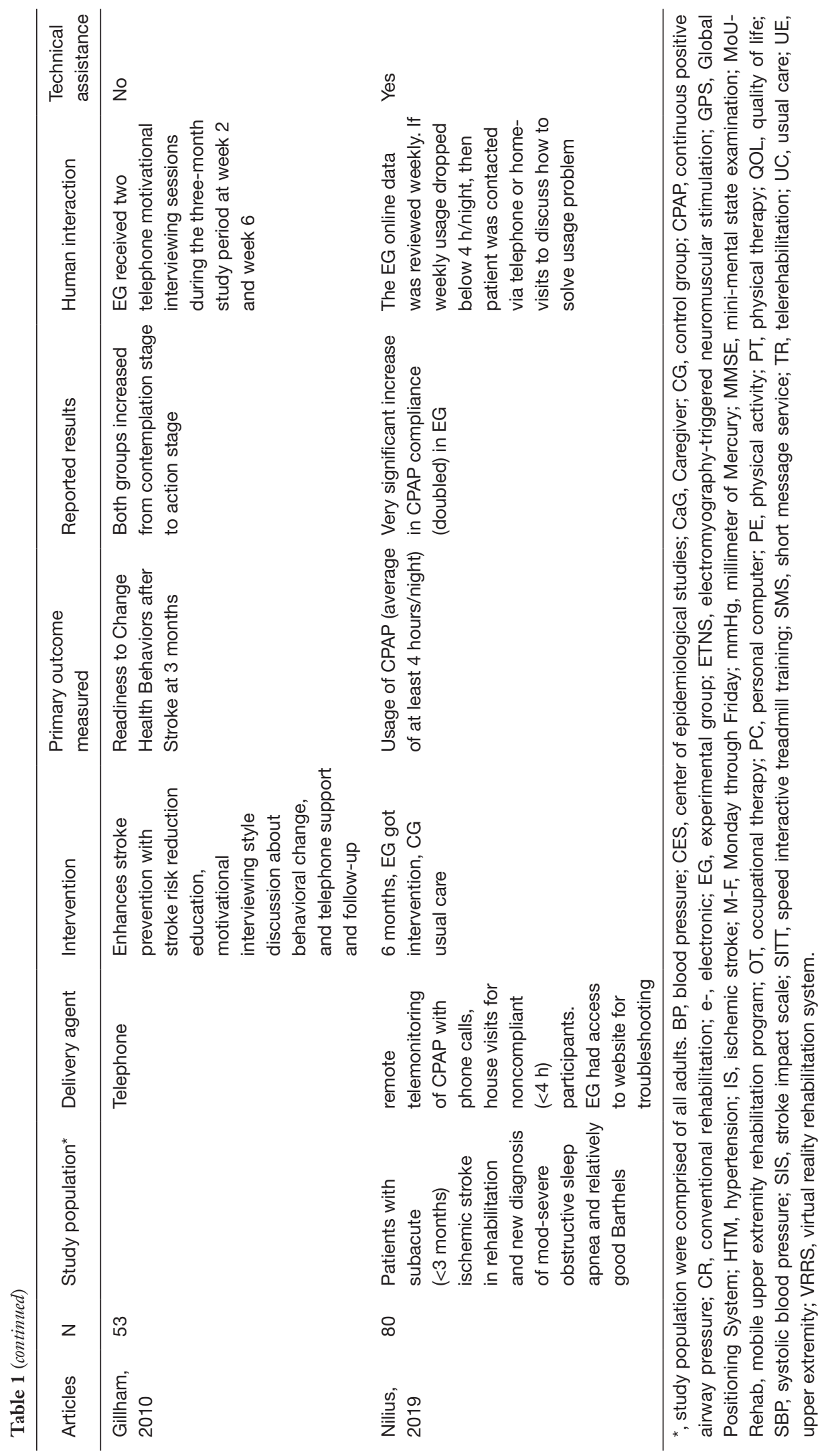


studies included using a novel $\mathrm{mHealth}$ application designed for the current study $(27,28,30,32,39)$. Fewer studies used SMS or other bidirectional communication applications $(31,32,35-37)$. A few studies used video as a means of patient monitoring and bidirectional communication $(29,30,32,40)$. Other sources of intervention delivery were through remote patient metric monitoring $(21,29,33)$, telerehabilitation delivery device $(24,28)$, and patient or provider education delivery $(34,36,38)$. The broad spectrum of intervention employed illustrates the complexity of conducting mHealth research among stroke populations.

QOL and daily functioning are often identified as high-priority outcomes among stroke survivors $(49,50)$. mHealth applications may be a reasonable tool to achieve positive QOL assessments as all included studies with QOL endpoints reported improvements in the participants receiving the active mHealth intervention. These findings must be balanced with the subjective nature of the assessments and the inability to blind participants, which both introduce response bias, and may reflect the overall beneficial effect of merely engaging a patient around their health in any form.

Durability and usability of any intervention are critical components of its effectiveness. The behavioral changes necessary to improve vascular risk factor management require motivation, a factor even more vulnerable to waning engagement. Long-term outcomes, therefore, will be important to demonstrate continued success of any given mHealth intervention and should be incorporated in study design. The studies reported in this review did not address any potential durability of any mHealth intervention as the assessments occurred over a range of two weeks to 12 months, with a median of only nine weeks. No study reported follow-up beyond the immediate intervention period. Furthermore, most studies occurred in the chronic phase ( $>6$ months from stroke event), well after the highest risk of stroke recurrence period has passed (1). All studies reported minimal issues related to usability. However, there is a selection bias considering that only patients who could operate the technology were included, and more than half of the studies (10) included some type of troubleshooting mechanism or periodic feasibility check-up. Apart from providing technical assistance, no study attempted to determine the kind or frequency of study participant engagement associated with a successful outcome.

While still developing, the mHealth literature investigating the management of isolated vascular risk factors, such as smoking cessation, hypertension, or glycemic control, among populations with these conditions is more robust (51-53). mHealth has been proven to be an effective strategy to reduce glycosylated hemoglobin and improve smoking cessation or systolic blood pressure $(54,55)$. However, it remains to be seen whether we can apply those results to a stroke patient who also has diabetes. Stroke is a multifactorial cumulative event; thus, reducing its recurrence is a complex proposition. Stroke survivors represent a heterogenous and clinically unique population, further emphasizing the difficulty of applying evidence derived from more narrowly defined clinical populations. Considering the complicated nature of the average stroke survivor, involving end-users in the development of a successful mHealth intervention to reduce recurrent stroke has been recommended, although rarely adopted (56-59).

The dearth of included studies as well as the incipient mHealth literature in general begs the question as to why mHealth remains understudied. Mobile technology has advanced rapidly, and its relative affordability has made it widely accessible to industry and the general public. Our ubiquitous reliance on cellular technology may have promoted the adoption of mHealth strategies at a rate faster than rigorous studies can be completed and published. In addition, the design of such studies is complex as they require tackling multiple vascular risk factors simultaneously $(60,61)$. Other critical factors a successful mHealth intervention may need to address are low stroke health literacy and navigating the increasingly complicated post-acute healthcare landscape. Recruitment efforts may also be hampered by discouraging stroke survivors with little familiarity of the proposed technology or those who lack internet access. Limited broadband coverage has been proven to contribute to rural/urban health disparities and a decrease in mHealth use (62).

There are limitations to this qualitative review, and no secondary qualitative analysis was performed. The studies discussed were diverse in primary purpose and outcomes. This scoping review also did not review literature that included dissertations, recommendations, or conference proceedings, which could introduce bias as only peerreviewed, published RCTs were included. Therefore, this study is vulnerable to possibly missing useful findings reported in other formats or unreported results (e.g., RCTs demonstrating the null hypothesis). Overall, however, our approach is considered rigorous given that we only included RCTs, with their associated risk of bias being low. Given the nature of the intervention, subject blinding was not feasible. Nine studies identified subjective outcomes 
(i.e., self-reported QOL, depression or functional status scores), potentially introducing performance bias. Enrolled populations were limited to those who could engage with and had access to the technology, creating a selection bias common to any implementation of mHealth. These latter factors limit the generalizability of any given mHealth intervention among a broader stroke population.

In summary, little high-quality evidence was found supporting mHealth that targeted stroke patients. Studies were heterogenous, lacked longitudinal follow-up, and involved a relatively small number of participants. While the majority of studies achieved their primary or secondary outcomes, many were purely subjective, and no single study identified secondary stroke prevention rate as the outcome measure of interest. Although mHealth may still prove to be a powerful way to address other clinically relevant targets (mood, daily living or functional outcomes), this lack of emphasis on secondary stroke prevention lays bare the gap in evidence addressing this population.

Consequently, systematically developing effective mHealth interventions is vital to harness the potential benefits of mobile technology as a healthcare surrogate. This review illustrates that mHealth for secondary stroke prevention remains understudied and also supports the critical need to design and complete RCTs utilizing different mHealth platforms with the specific aim to decrease recurrent stroke rates, especially in the highest risk period ( $<3$ months post-stroke). If an appropriate tool or tools can be designed, tested, and implemented, the potential to impact stroke care and chronic disease management in general is immense.

\section{Acknowledgments}

Funding: The project described was supported by the National Institute of General Medical Sciences, U54GM104942. The content is solely the responsibility of the authors and does not necessarily represent the official views of the NIH.

\section{Footnote}

Reporting Checklist: The authors have completed the PRISMAScR reporting checklist. Available at https://mhealth. amegroups.com/article/view/10.21037/mhealth-21-27/rc

Peer Review File: Available at https://mhealth.amegroups. com/article/view/10.21037/mhealth-21-27/prf
Conflicts of Interest: All authors have completed the ICMJE uniform disclosure form (available at https://mhealth. amegroups.com/article/view/10.21037/mhealth-21-27/ coif). The authors have no conflicts of interest to declare.

Ethical Statement: The authors are accountable for all aspects of the work in ensuring that questions related to the accuracy or integrity of any part of the work are appropriately investigated and resolved.

Open Access Statement: This is an Open Access article distributed in accordance with the Creative Commons Attribution-NonCommercial-NoDerivs 4.0 International License (CC BY-NC-ND 4.0), which permits the noncommercial replication and distribution of the article with the strict proviso that no changes or edits are made and the original work is properly cited (including links to both the formal publication through the relevant DOI and the license). See: https://creativecommons.org/licenses/by-nc-nd/4.0/.

\section{References}

1. Oza R, Rundell K, Garcellano M. Recurrent Ischemic Stroke: Strategies for Prevention. Am Fam Physician 2017;96:436-40.

2. Redfern J, McKevitt C, Dundas R, et al. Behavioral risk factor prevalence and lifestyle change after stroke: a prospective study. Stroke 2000;31:1877-81.

3. Hackam DG, Spence JD. Combining multiple approaches for the secondary prevention of vascular events after stroke: a quantitative modeling study. Stroke 2007;38:1881-5.

4. Lovett JK, Coull AJ, Rothwell PM. Early risk of recurrence by subtype of ischemic stroke in populationbased incidence studies. Neurology 2004;62:569-73.

5. O'Donnell MJ, Xavier D, Liu L, et al. Risk factors for ischaemic and intracerebral haemorrhagic stroke in 22 countries (the INTERSTROKE study): a case-control study. Lancet 2010;376:112-23.

6. Williams B, Masi S, Wolf J, et al. Facing the Challenge of Lowering Blood Pressure and Cholesterol in the Same Patient: Report of a Symposium at the European Society of Hypertension. Cardiol Ther 2020;9:19-34.

7. Hall J, Morton S, Fitzsimons CF, et al. Factors influencing sedentary behaviours after stroke: findings from qualitative observations and interviews with stroke survivors and their caregivers. BMC Public Health 2020;20:967.

8. Grau-Pellicer M, Chamarro-Lusar A, Medina-Casanovas J, et al. Walking speed as a predictor of community mobility 
and quality of life after stroke. Top Stroke Rehabil 2019;26:349-58.

9. Allen MS, Walter EE, Swann C. Sedentary behaviour and risk of anxiety: A systematic review and meta-analysis. J Affect Disord 2019;242:5-13.

10. English C, Healy GN, Coates A, et al. Sitting and Activity Time in People With Stroke. Phys Ther 2016;96:193-201.

11. Lowe DB, Sharma AK, Leathley MJ. The CareFile Project: a feasibility study to examine the effects of an individualised information booklet on patients after stroke. Age Ageing 2007;36:83-9.

12. Kim AS. Medical Management for Secondary Stroke Prevention. Continuum (Minneap Minn) 2020;26:435-56.

13. Pfaeffli Dale L, Dobson R, Whittaker R, et al. The effectiveness of mobile-health behaviour change interventions for cardiovascular disease self-management: A systematic review. Eur J Prev Cardiol 2016;23:801-17.

14. Griffin LJ, Hickey JV. Considerations and strategies for educating stroke patients with neurological deficits. Journal of Nursing Education and Practice 2013;3:125.

15. Labrique AB, Vasudevan L, Kochi E, et al. mHealth innovations as health system strengthening tools: 12 common applications and a visual framework. Glob Health Sci Pract 2013;1:160-71.

16. Park YT. Emerging New Era of Mobile Health Technologies. Healthc Inform Res 2016;22:253-4.

17. Mamlin BW, Tierney WM. The Promise of Information and Communication Technology in Healthcare: Extracting Value From the Chaos. Am J Med Sci 2016;351:59-68.

18. Fiordelli M, Diviani N, Schulz PJ. Mapping mHealth research: a decade of evolution. J Med Internet Res 2013;15:e95.

19. Jansons PS, Robins L, Haines TP, et al. Barriers and enablers to ongoing exercise for people with chronic health conditions: Participants' perspectives following a randomized controlled trial of two interventions. Arch Gerontol Geriatr 2018;76:92-9.

20. Weerahandi H, Paul S, Quintiliani LM, et al. A Mobile Health Coaching Intervention for Controlling Hypertension: Single-Arm Pilot Pre-Post Study. JMIR Form Res 2020;4:e13989.

21. Paul L, Wyke S, Brewster S, et al. Increasing physical activity in stroke survivors using STARFISH, an interactive mobile phone application: a pilot study. Top Stroke Rehabil 2016;23:170-7.

22. Bhavnani SP, Narula J, Sengupta PP. Mobile technology and the digitization of healthcare. Eur Heart J 2016;37:1428-38.
23. Higgins JP, Altman DG, Gøtzsche PC, et al. The Cochrane Collaboration's tool for assessing risk of bias in randomised trials. BMJ 2011;343:d5928.

24. Chen J, Jin W, Dong WS, et al. Effects of Home-based Telesupervising Rehabilitation on Physical Function for Stroke Survivors with Hemiplegia: A Randomized Controlled Trial. Am J Phys Med Rehabil 2017;96:152-60.

25. Choi YH, Ku J, Lim H, et al. Mobile game-based virtual reality rehabilitation program for upper limb dysfunction after ischemic stroke. Restor Neurol Neurosci 2016;34:455-63.

26. van den Berg M, Crotty M Prof, Liu E, et al. Early Supported Discharge by Caregiver-Mediated Exercises and e-Health Support After Stroke: A Proof-of-Concept Trial. Stroke 2016;47:1885-92.

27. Kang YN, Shen HN, Lin CY, et al. Does a Mobile app improve patients' knowledge of stroke risk factors and health-related quality of life in patients with stroke? A randomized controlled trial. BMC Med Inform Decis Mak 2019;19:282.

28. Linder SM, Rosenfeldt AB, Bay RC, et al. Improving Quality of Life and Depression After Stroke Through Telerehabilitation. Am J Occup Ther 2015;69:6902290020p1-10.

29. Nilius G, Schroeder M, Domanski U, et al. Telemedicine Improves Continuous Positive Airway Pressure Adherence in Stroke Patients with Obstructive Sleep Apnea in a Randomized Trial. Respiration 2019;98:410-20.

30. Torrisi M, Maresca G, De Cola MC, et al. Using telerehabilitation to improve cognitive function in poststroke survivors: is this the time for the continuity of care? Int J Rehabil Res 2019;42:344-51.

31. Sarfo FS, Treiber F, Gebregziabher M, et al. Phone-based intervention for blood pressure control among Ghanaian stroke survivors: A pilot randomized controlled trial. Int J Stroke 2019;14:630-8.

32. Chumbler NR, Li X, Quigley P, et al. A randomized controlled trial on Stroke telerehabilitation: The effects on falls self-efficacy and satisfaction with care. J Telemed Telecare 2015;21:139-43.

33. Grau-Pellicer M, Lalanza JF, Jovell-Fernández E, et al. Impact of mHealth technology on adherence to healthy PA after stroke: a randomized study. Top Stroke Rehabil 2020;27:354-68.

34. Gillham S, Endacott R. Impact of enhanced secondary prevention on health behaviour in patients following minor stroke and transient ischaemic attack: a randomized controlled trial. Clin Rehabil 2010;24:822-30. 
35. Kamal AK, Shaikh Q, Pasha O, et al. A randomized controlled behavioral intervention trial to improve medication adherence in adult stroke patients with prescription tailored Short Messaging Service (SMS)SMS4Stroke study. BMC Neurol 2015;15:212.

36. Cadilhac DA, Andrew NE, Busingye D, et al. Pilot randomised clinical trial of an eHealth, self-management support intervention (iVERVE) for stroke: feasibility assessment in survivors 12-24 months post-event. Pilot Feasibility Stud 2020;6:172.

37. Owolabi MO, Gebregziabher M, Akinyemi RO, et al. Randomized Trial of an Intervention to Improve Blood Pressure Control in Stroke Survivors. Circ Cardiovasc Qual Outcomes 2019;12:e005904.

38. Smith GC, Egbert N, Dellman-Jenkins M, et al. Reducing depression in stroke survivors and their informal caregivers: a randomized clinical trial of a Web-based intervention. Rehabil Psychol 2012;57:196-206.

39. Lee J, Lee K, Song C. Speed-Interactive Treadmill Training Using Smartphone-Based Motion Tracking Technology Improves Gait in Stroke Patients. J Mot Behav 2017;49:675-85.

40. Lin KH, Chen CH, Chen YY, et al. Bidirectional and multi-user telerehabilitation system: clinical effect on balance, functional activity, and satisfaction in patients with chronic stroke living in long-term care facilities. Sensors (Basel) 2014;14:12451-66.

41. CDC Wonder 2021. Centers for Disease Control. Census Population Projections. [April 2021]. Available online: https://wonder.cdc.gov/population.html

42. Liu S, Feng W, Chhatbar PY, et al. Mobile health as a viable strategy to enhance stroke risk factor control: A systematic review and meta-analysis. J Neurol Sci 2017;378:140-5.

43. Fruhwirth V, Enzinger C, Weiss E, et al. Use of smartphone apps in secondary stroke prevention. Wien Med Wochenschr 2020;170:41-54.

44. Bartoli F, Lillia N, Lax A, et al. Depression after stroke and risk of mortality: a systematic review and meta-analysis. Stroke Res Treat 2013;2013:862978.

45. Espárrago Llorca G, Castilla-Guerra L, Fernández Moreno MC, et al. Post-stroke depression: an update. Neurologia 2015;30:23-31.

46. Whooley MA. Depression and cardiovascular disease: healing the broken-hearted. JAMA 2006;295:2874-81.

47. Kronenberg G, Gertz K, Heinz A, et al. Of mice and men: modelling post-stroke depression experimentally. Br J Pharmacol 2014;171:4673-89.
48. Nakase T, Tobisawa M, Sasaki M, et al. Outstanding Symptoms of Poststroke Depression during the Acute Phase of Stroke. PLoS One 2016;11:e0163038.

49. Franceschini M, La Porta F, Agosti M, et al. Is healthrelated-quality of life of stroke patients influenced by neurological impairments at one year after stroke? Eur J Phys Rehabil Med 2010;46:389-99.

50. Patel MD, McKevitt C, Lawrence E, et al. Clinical determinants of long-term quality of life after stroke. Age Ageing 2007;36:316-22.

51. Cramm JM, Nieboer AP. A longitudinal study to identify the influence of quality of chronic care delivery on productive interactions between patients and (teams of) healthcare professionals within disease management programmes. BMJ Open 2014;4:e005914.

52. Gagnon MP, Ndiaye MA, Larouche A, et al. Optimising patient active role with a user-centred eHealth platform (CONCERTO+) in chronic diseases management: a study protocol for a pilot cluster randomised controlled trial. BMJ Open 2019;9:e028554.

53. Marcolino MS, Oliveira JAQ, D'Agostino M, et al. The Impact of mHealth Interventions: Systematic Review of Systematic Reviews. JMIR Mhealth Uhealth 2018;6:e23.

54. Wayne N, Perez DF, Kaplan DM, et al. Health Coaching Reduces HbA1c in Type 2 Diabetic Patients From a Lower-Socioeconomic Status Community: A Randomized Controlled Trial. J Med Internet Res 2015;17:e224.

55. Ali MK, Chwastiak L, Poongothai S, et al. Effect of a Collaborative Care Model on Depressive Symptoms and Glycated Hemoglobin, Blood Pressure, and Serum Cholesterol Among Patients With Depression and Diabetes in India: The INDEPENDENT Randomized Clinical Trial. JAMA 2020;324:651-62.

56. Peng W, Yuan S, Holtz BE. Exploring the Challenges and Opportunities of Health Mobile Apps for Individuals with Type 2 Diabetes Living in Rural Communities. Telemed J E Health 2016;22:733-8.

57. Towfighi A, Cheng EM, Ayala-Rivera M, et al. Randomized controlled trial of a coordinated care intervention to improve risk factor control after stroke or transient ischemic attack in the safety net: Secondary stroke prevention by Uniting Community and Chronic care model teams Early to End Disparities (SUCCEED). BMC Neurol 2017;17:24.

58. McCurdie T, Taneva S, Casselman M, et al. mHealth consumer apps: the case for user-centered design. Biomed Instrum Technol 2012;Suppl:49-56.

59. Zhang H, Wang T, Zhang Z, et al. The current status of 
stroke-related smartphone applications available to adopt in China: A systematic review study. Medicine (Baltimore) 2020;99:e20656.

60. Piette JD, List J, Rana GK, et al. Mobile Health Devices as Tools for Worldwide Cardiovascular Risk Reduction and Disease Management. Circulation 2015;132:2012-27. 61. Whitehead L, Seaton P. The Effectiveness of Self-

\section{doi: $10.21037 /$ mhealth-21-27}

Cite this article as: Adcock AK, Haggerty T, Crawford A, Espinosa C. mHealth impact on secondary stroke prevention: a scoping review of randomized controlled trials among stroke survivors between 2010-2020. mHealth 2022;8:19.
Management Mobile Phone and Tablet Apps in Longterm Condition Management: A Systematic Review. J Med Internet Res 2016;18:e97.

62. Giacobbi P Jr, Cushing P, Popa A, et al. Mobile Health (mHealth) Use or Non-Use by Residents of West Virginia. South Med J 2018;111:625-7. 
mHealth and secondary stroke prevention

mHealth Concept: included in search

mHealth

mobile health

telehealth

telemedicine

eHealth

mobile app*

smartphone app*

phone app*

"Mobile Applications"[Mesh] "Telemedicine"[Mesh]

"Smartphone"[Mesh]

"Cell Phone"[Mesh]

Secondary stroke concept: included in search

secondary stroke *

recurrent stroke*

recurring stroke*

stroke recurrence

secondary prevention adj3 stroke

"Stroke Rehabilitation"[Mesh]

(“Secondary Prevention”[Mesh] AND "Stroke”[Mesh])

Search Strategies

Run in Ovid Medline, Cochrane Library, Scopus and CINAHL

Total results: 481

Duplicates found in Endnote: 129

Results after deduplication: 352

Ovid Medline

Run $11 / 19 / 2020$

Results 312 


\begin{tabular}{|c|c|c|}
\hline & Searches & Results \\
\hline 1 & mHealth.mp. & 5,015 \\
\hline 2 & mobile health.mp. & 8,161 \\
\hline 3 & telehealth.mp. & 5,411 \\
\hline 4 & telemedicine.mp. & 30,363 \\
\hline 5 & eHealth.mp. & 4,107 \\
\hline 6 & mobile app*.mp. & 9,726 \\
\hline 7 & smartphone app*.mp. & 3,358 \\
\hline 8 & phone app*.mp. & 1,138 \\
\hline 9 & exp Mobile Applications/ & 6,529 \\
\hline 10 & exp Telemedicine/ & 30,836 \\
\hline 11 & exp Cell Phone/ & 11,043 \\
\hline 12 & exp Smartphone/ & 4,093 \\
\hline 13 & or/1-12 & 64,734 \\
\hline 14 & secondary stroke*.mp. & 1,198 \\
\hline 15 & recurrent stroke*.mp. & 3,598 \\
\hline 16 & recurring stroke*.mp. & 21 \\
\hline 17 & stroke recurrence.mp. & 1,573 \\
\hline 18 & (secondary prevention adj3 stroke).mp. & 1,158 \\
\hline 19 & exp Secondary Prevention/ & 20,707 \\
\hline 20 & exp Stroke/ & 137,964 \\
\hline 21 & 19 and 20 & 2,028 \\
\hline 22 & exp Stroke Rehabilitation/ & 13,666 \\
\hline 23 & or/14-18,21-22 & 20,776 \\
\hline 24 & 13 and 23 & 312 \\
\hline
\end{tabular}


Cochrane Library

Run $11 / 19 / 2020$

Results 84

ID Search Hits

1 (mHealth):ti,ab,kw 1190

2 (“mobile health"):ti,ab,kw 1109

3 (telehealth):ti,ab,kw 1706

4 (telemedicine):ti,ab,kw 3683

5 (eHealth):ti,ab,kw 919

6 (“mobile app*”):ti,ab,kw 692

7 (“smartphone app"”):ti,ab,kw 610

8 ("phone app*"):ti,ab,kw 197

9 MeSH descriptor: [Mobile Applications] explode all trees 640

$10 \mathrm{MeSH}$ descriptor: [Telemedicine] explode all trees 2547

$11 \mathrm{MeSH}$ descriptor: [Cell Phone] explode all trees 1354

$12 \mathrm{MeSH}$ descriptor: [Smartphone] explode all trees 397

13 \#1 OR \#2 OR \#3 OR \#4 OR \#5 OR \#6 OR \#7 OR \#8 OR \#9 OR \#10 OR \#11 OR \#129782

14 (“secondary stroke"):ti,ab,kw 363

15 ("recurrent stroke"):ti,ab,kw 1030

16 (“recurring stroke*"):ti,ab,kw 4

17 ("stroke recurrence"):ti,ab,kw 377

18 ((“secondary prevention” NEAR/3 stroke)):ti,ab,kw 418

$19 \mathrm{MeSH}$ descriptor: [Secondary Prevention] explode all trees 3156

$20 \mathrm{MeSH}$ descriptor: [Stroke] explode all trees $\quad 9802$

21 \#19 AND \#20 331

$22 \mathrm{MeSH}$ descriptor: [Stroke Rehabilitation] explode all trees 2442

23 \#14 OR \#15 OR \#16 OR \#17 OR \#18 OR \#21 OR \#22 4379

24\#13 AND \#23 84

Scopus

Run $11 / 19 / 2020$

Results 60

((TITLE-ABS-KEY (mhealth) OR TITLE-ABS-KEY (“mobile health”) OR TITLE-ABS-KEY (telehealth) OR TITLEABS-KEY (telemedicine) OR TITLE-ABS-KEY (ehealth) OR TITLE-ABS-KEY (“mobile app*”) OR TITLE-ABS-KEY (“smartphone app*”) OR TITLE-ABS-KEY (“phone app*”))) AND ((TITLE-ABS-KEY (“secondary stroke*”) OR TITLEABS-KEY ("recurrent stroke*”) OR TITLE-ABS-KEY (“recurring stroke*”) OR TITLE-ABS-KEY (“stroke recurrence”) OR TITLE-ABS-KEY (“secondary prevention” W/3 stroke))) 
CINAHL

Run 11/20/2020

Results 25

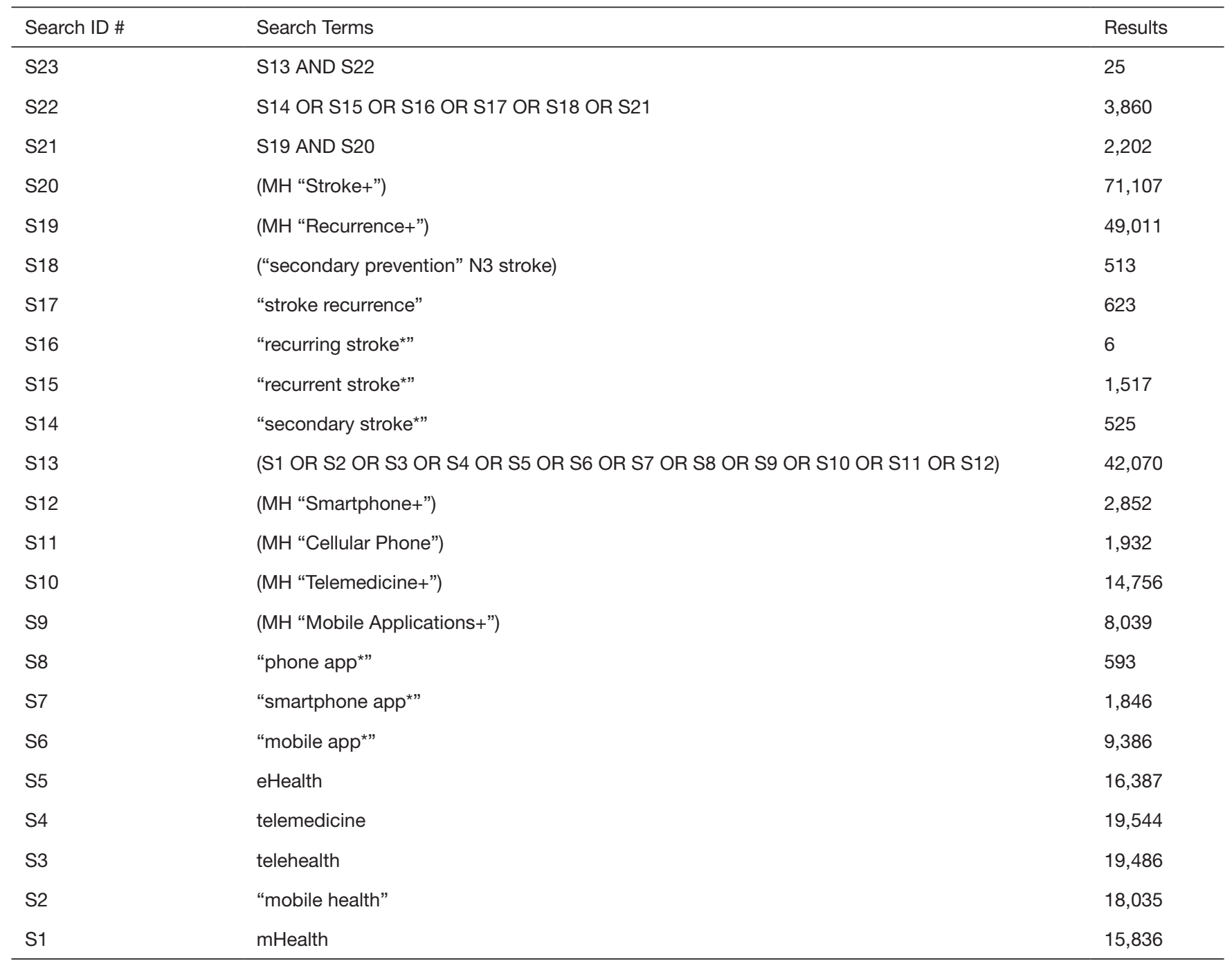

